\title{
Review
}

\section{Role of Magnetic Resonance Imaging in Visualizing Coronary Arteries}

\author{
Sumeesh Dhawan MD, Department of Internal Medicine, Marshfield Clinic, Marshfield, Wisconsin \\ Kodlipet C. Dharmashankar, MD, Department of Internal Medicine, Marshfield Clinic, Marshfield, Wisconsin \\ Tahir Tak MD, PhD*, Department of Cardiology, Marshfield Clinic, Marshfield, Wisconsin \\ ${ }^{*}$ Current affiliation: Division of Cardiology, Department of Internal Medicine, University of North Texas Health Science Center, Fort Worth, Texas
}

\begin{abstract}
Ischemic heart disease is the leading cause of death worldwide. At present, coronary angiography is the gold standard for the diagnosis of coronary artery disease. Conventional coronary angiography is an invasive procedure with a small, yet inherent risk of myocardial infarction, stroke, potential arrhythmias, and death. Other noninvasive diagnostic tools, such as electrocardiography, echocardiography, and nuclear imaging are now widely available but are limited by their inability to directly visualize and quantify coronary artery stenoses and predict the stability of plaques. Coronary magnetic resonance angiography (MRA) is a technique that allows visualization of the coronary arteries by noninvasive means; however, it has not yet reached a stage where it can be used in routine clinical practice. Although coronary MRA is a potentially useful diagnostic tool, it has limitations. Further research should focus on improving the diagnostic resolution and accuracy of coronary MRA. This review summarizes results from several studies comparing coronary MRA with conventional coronary angiography. Current two-dimensional and three-dimensional coronary MRA techniques and their limitations are also discussed.
\end{abstract}

REPRINT REQUESTS:

Tahir Tak, MD, PhD

Division of Cardiology

Department of Internal Medicine

University of North Texas Health Science Center

855 Montgomery Street

Fort Worth, TX 76104

Telephone: 817-735-2332

Fax: 817-735-2673

Email: ttak@hsc.unt.edu

\section{KEYWORDS:}

Coronary vessels; Angiography;

Imaging, magnetic resonance 


\section{INTRODUCTION}

Ischemic heart disease is the leading cause of death worldwide. ${ }^{1}$ For patients with coronary artery disease (CAD), treatment consists of medical therapy, percutaneous intervention, or coronary artery bypass grafting. The most essential diagnostic tool to evaluate graft disease is coronary angiography. This technique provides rapid and accurate delineation of the entire coronary anatomy including the graft, and the native non-grafted coronary arteries, and it is generally used as a prelude to revascularization procedures.

Coronary magnetic resonance angiography (MRA) is a technique that allows the visualization of coronary arteries by non-invasive means. Since it was first reported by Paulin et al. ${ }^{2}$ in 1987, coronary MRA has gained considerable importance as a method that could be used to diagnose coronary artery stenoses. The potential benefit of coronary MRA is not only the visualization of coronary arteries, but also the visualization of the cardiac morphology, acquired diseases of the great vessels, and cardiac function at rest and under stress. ${ }^{3-5}$ Other benefits of MRA include assessment of the patency of aorto-coronary bypass grafts, anomalous coronary arteries, ${ }^{6}$ and possible stability of plaques without significant exposure of ionizing radiation. ${ }^{7,8}$ However, further studies are needed to corroborate these findings.

Conventional coronary angiography is considered the gold standard for the visualization of CAD. Nevertheless, conventional coronary angiography is an invasive procedure. It involves a small but significant risk of acute myocardial infarction, stroke, and potential arrhythmias. ${ }^{9}$ Other technologies are limited by their inability to directly visualize and quantify coronary artery stenoses. ${ }^{10}$ Non-invasive diagnostic tools such as electrocardiography, echocardiography, and nuclear imaging are also widely available, and are useful in analyzing underlying CAD that has progressed to a significant level. However, these techniques cannot predict the stability of plaques. Coronary MRA is a non-invasive technique that not only helps in detection of CAD at an early stage but also predicts the stability of plaques. Coronary MRA has the potential to become a useful screening tool for patients with atypical symptoms of myocardial ischemia, ${ }^{10}$ and would help in the initiation of primary preventive measures at an earlier stage. ${ }^{10}$

Various studies comparing the results of coronary MRA imaging with conventional coronary angiography are discussed in this review. A formal meta-analysis was not performed because of the different techniques involved in the studies, the heterogeneity of study designs, and the small number of patients involved in these studies.

\section{CURRENT CORONARY MRA TECHNIQUES AND TECHNICAL LIMITATIONS}

Coronary MRA has evolved as a promising non-invasive modality for the imaging of the coronary arteries. It is also useful in the evaluation of coronary artery stenoses.

Images obtained during coronary MRA can be visualized in 2-dimensional (2D) or 3-dimensional (3D) views. The cardiac contractions and the motion of the diaphragm limit the quality of images. Different techniques, such as a breath hold technique versus a free breathing with navigator echo-based technique, have been employed to overcome these limitations. For a 2D coronary MRA utilizing the breath hold technique, the patient may be required to do 30 or more breath holds for 16-20 seconds, and may be even longer for a 3D coronary MRA. ${ }^{11,12}$ On the other hand, the free breathing with navigator technique enables respiratory synchronization of image acquisition to a specific phase and spatial location of the diaphragm with prospective or retrospective gating (figure 1). ${ }^{11,12}$

Two main approaches have been developed to visualize the endovascular lumen. In the conventional spin-echo approach, the areas of the vessel with turbulent flow (non-stenosed segments) appear black, whereas in the commonly used gradient-echo approach, the vessels with laminar flow appear dark in color, and those with turbulent flow secondary to stenoses appear bright. ${ }^{10-12}$ Other technical impediments include a relatively small coronary artery diameter ( 2.7 to $3.5 \mathrm{~mm}$ ), ${ }^{13}$ tortuous course of the vessel, and the surrounding epicardial fat. Furthermore, the interpretation of the imaging of a left circumflex coronary artery in MRA is limited by difficulty to distinguish the artery from parallel running cardiac veins. ${ }^{12,13}$

Several studies have assessed the diagnostic accuracy of 2D and $3 \mathrm{D}$ coronary MRA in comparison to conventional coronary angiography. Although not yet standardized, the current method for coronary MRA combines fast imaging 3D techniques with respiratory gated coronary MRA using navigator echo that improves its diagnostic accuracy. The navigator echo technique seems to have more application in clinical practice, as the breath hold technique is not always possible for patients with congestive heart failure, $\mathrm{CAD}$, and chronic obstructive pulmonary disease. ${ }^{11,12}$ However, in those cases the quality of images may be degraded due to inconsistent breathing patterns and patient movements. ${ }^{14}$

The other alternative techniques include magnetic resonance subtraction methods, which include selective tagging of blood in the aortic root and suppression of the background tissue. This method holds potential for visualization of the proximal portion of the coronary artery, but the length of the vessel can only be visualized if there is blood flow throughout the coronary artery. Thus, if the blood flow is impaired in the presence of coronary artery stenoses, visualization of the entire artery is not possible. Other techniques, such as spiral acquisition echo planar imaging 15 and segment echo planar imaging techniques, ${ }^{16}$ provide a complete 3D data set encompassing the entire heart and can be acquired in one or two breath holds. 

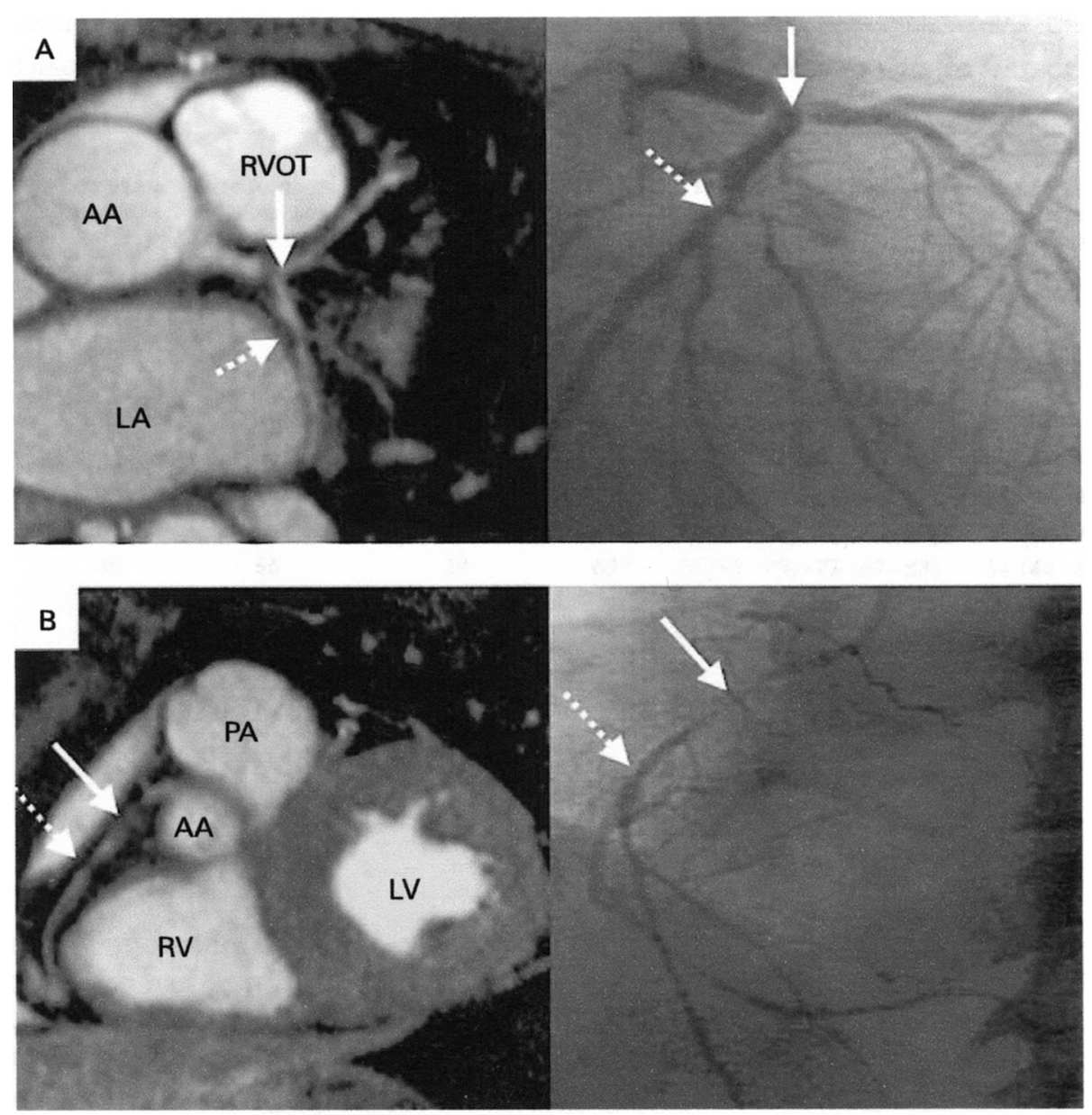

Figure 1. Coronary angiography in a 53-year-old man with exertional chest pain. Panel A shows a coronary magnetic resonance angiogram (left) and a corresponding $\mathrm{x}$-ray coronary angiogram (right) indicating a severe lesion at the bifurcation of the left anterior descending coronary artery and the left circumflex coronary artery, involving the left main coronary artery (solid arrows), and a more distal focal stenosis of the left circumflex coronary artery (broken arrows). Panel B shows a coronary magnetic resonance angiogram (left) and a corresponding x-ray angiogram (right) indicating two stenoses of the proximal (solid arrows) and middle (broken arrows) right coronary artery. AA denotes ascending aorta, LA left atrium, RVOT right ventricular outflow tract, PA pulmonary artery, RV right ventricle, and LV left ventricle. (Reprinted with permission. $\mathrm{Kim}$ et al. Coronary magnetic resonance angiography for the detection of coronary stenoses. N Engl J Med 2001;345:1863-1969. Copyright 2001 Massachusetts Medical Society. All rights reserved.)

\section{TWO-DIMENSIONAL CORONARY MRA}

Edelman et al. ${ }^{17}$ published the first report of a clinical application of 2D coronary MRA to visualize the coronary arteries in 1991, though the first comparison of the results of 2D coronary MRA with conventional coronary angiography was done by Manning et al. ${ }^{18}$ in 1993. Overall, the sensitivity and specificity of the 2D coronary MRA technique for correctly identifying the significant stenoses in the coronary artery $(>50 \%)$ based upon coronary angiography was $90 \%$ and $92 \%$, respectively. Studies by Duerinckx and Urman, ${ }^{19}$ Pennell et al., ${ }^{20}$ and Post et al. ${ }^{21}$ failed to reproduce Manning's results.

One of the few studies in which a large number of patients were evaluated with 2D coronary MRA utilizing the breath hold technique was performed by Watanuki et al. ${ }^{22}$ involving 108 patients. When compared with coronary angiography, this technique was very sensitive (85\%) and specific $(80 \%)$ for diagnosing severe coronary artery stenoses $(90 \%$ to $100 \%$ ), but for moderate stenoses (more than $50 \%$ ), the sensitivity was as low as $38 \%$, while the specificity was $83 \%$. The low sensitivity was attributed to the method of detecting the stenoses. The detection of stenoses was dependent upon a decrease in the signal intensity secondary to a decrease in the blood flow. The rate of blood flow can be significantly affected by the tortuous course of the artery, as well as the characteristics of stenoses. The quality of images was distorted by the inability of the patient to hold his or her breath for 15 seconds and/or the slight movements of the diaphragm.

It should be noted that 2D gradient-echo breath-hold coronary MRA has several drawbacks:

1. Relatively thick sections (4-6 mm in most studies) may preclude an exact grading of focal stenoses. ${ }^{18,20}$ This probably also explains the limited evaluation of tortuous vessels and the distal portions of major coronary arteries, especially the left circumflex coronary artery. ${ }^{20,23,24}$

2. Different oblique planes are required for different patients and for the visualization of different arteries. ${ }^{23,24}$

3. Multiple breath holds can be a practical problem in certain patient populations with a history of congestive heart failure, chronic obstructive pulmonary disease, and severe CAD. ${ }^{11,12}$

4. Inconsistent breath-holds and misregistration between breath holds may lead to inadequate vessel assessment. ${ }^{24-26}$

\section{THREE-DIMENSIONAL CORONARY MRA}

The first 3D coronary MRAs were used by Paschal et al. ${ }^{27}$ and $\mathrm{Li}$ et al. ${ }^{28}$ in 1993 . However, these studies did not employ the use of the navigator-based echo technique; they used the method of averaging multiple acquisitions without respiratory synchronization. Significant blurring has been reported with those techniques..$^{29}$ Hofman et al. ${ }^{30}$ used the navigator echo techniques for the first time and compared 
the results of $2 \mathrm{D}$ breath holding with $3 \mathrm{D}$ respiratory gating technique. Post et al. ${ }^{31}$ studied 20 patients who had undergone respiratory gated 3D coronary MRA and conventional coronary angiography. The studies concluded that even though these techniques provide a good view of proximal, as well as middle segments of the coronary arteries, the sensitivity for detecting coronary artery stenoses was decreased to $38 \%$, however, the specificity remained as high as $95 \%$. The low sensitivity was likely due to the differences in the techniques relating to relatively poor temporal resolution, shorter echo time, poor patient cooperation with breath holding, or irregular rhythms, all of which attribute to image degradation. ${ }^{12}$

The study by Kim et al. ${ }^{32}$ was the first multicenter trial where 109 subjects were evaluated for CAD by the free-breathing 3D technique. However, this technique was limited to evaluation of proximal and middle segments only. In this study a total of 636 of 759 proximal and middle segments of coronary arteries were interpretable. A sensitivity of $83 \%$ was reported for this technique. However, lesions in the left main coronary artery in patients with three vessel disease could be identified with some certainty in a limited number of patients. ${ }^{32}$ The study also stated that major limitations with the 3D technique are the relatively longer time (average 70 minutes), low specificity (42\%), and low overall diagnostic accuracy $(72 \%)$.

Muller et al. ${ }^{33}$ conducted a study in which 35 patients underwent coronary MRA with single slab, three dimensional gradient-echo sequences employing a spin-echo navigator echo measurement and conventional angiography. The sensitivity, specificity, and positive and negative predictive values of coronary MRA in detecting significant stenoses $(>50 \%)$ were $83 \%, 94 \%, 87 \%$, and $93 \%$, respectively. The high sensitivity and specificity reported from this study was never reproduced in other studies. A major limitation in this study was that only proximal and middle segments of the main coronary arteries were considered and the number of subjects was small. Kessler et al. ${ }^{34}$ conducted a similar study in 73 patients who had undergone cardiac catheterization as well as $3 \mathrm{D}$ respiratory-gated coronary MRA. The sensitivity and specificity for the diagnoses of stenoses in the proximal and middle coronary arteries was $65 \%$ and $88 \%$, respectively. Sandstede et al. ${ }^{35}$ reproduced similar results in a study of 30 patients determining the sensitivity and specificity of detecting coronary artery stenoses in the four main coronary arteries at $81 \%$ and $89 \%$, respectively. The small number of subjects and evaluation of only the proximal and middle portions of the coronary arteries were limitations of this study.

Sardenelli et al. ${ }^{36}$ tried to identify the sensitivity of 3D navigator echo coronary MRA in detecting stenoses of the coronary arteries in a group of 42 patients. They concluded that coronary MRA has a sensitivity of $82 \%$ and specificity of $89 \%$, and that $3 \mathrm{D}$ coronary MRA has increased sensitivity for the detection of stenoses in the proximal segment of the coronary artery. The sensitivity was $90 \%$ for proximal and middle segments, but dropped to $68 \%$ when distal segments were included.

Weber et al. ${ }^{37}$ conducted a blinded study of the respiratory motion compensated technique (motion-adapted gating) for visualization of the coronary arteries in 15 subjects (11 patients and 4 volunteers). ${ }^{10}$ Results revealed that the coronary MRA correlated with coronary angiography. They used the electrocardiography-triggered respiratory motion gated 3D turbo field echo sequence technique defining a sensitivity of $88 \%$ and a specificity of $94 \%$, with positive and negative predictive values of $83 \%$ and $96 \%$, respectively. However, the motion-adapted gating concept has substantial limitations. It assigns more importance to the contrast than to the edge definition. Edge definition and sharpness are the prerequisites for stenosis detection and quantification. This results in high false positive values (e.g., in this study, five patients and two volunteers received high false positive values) and still remains a technical problem to be solved.

Watanabe et al. ${ }^{38}$ performed a study involving 12 patients. All 12 patients had undergone conventional coronary angiography, as well as high resolution selective 3D coronary MRA by orienting the 3D slab along the major axis of the right coronary artery, left circumflex or left main, or the left anterior descending group. On retrospective analysis, the sensitivity, specificity, and accuracy of identification of significant coronary artery stenoses was $80 \%, 85 \%$, and $84 \%$, respectively. Even though high resolution selective coronary MRA can provide information on segmental anatomy in detail, this technique has low sensitivity for the left circumflex artery. The other major problem with this method is its lengthy examination time that may cause degradation in image quality due to body motion.

Two studies have been performed where the use of the 3D coronary MRA with free breathing respiratory gating technique was used with some other techniques. Regenfus et al. ${ }^{39}$ compared the results of the contrast enhanced breath holding technique with the free breathing respiratory gating technique in 38 patients. The sensitivity using the breath holding technique was $86.7 \%$, and significantly higher than the free breathing respiratory gating technique $(60 \%)$. The higher sensitivity of the breath holding technique than the free breathing respiratory gating technique can be attributed to improvement in the imaging quality secondary to the use of contrast media. Contrast enhanced image relies on anatomic enhancement of stenoses rather than on flow effect. ${ }^{39,40}$ Moreover, it can be assumed that respiratory gating may be an ineffective technique in improving image quality.

Nikolaou et al. ${ }^{41}$ studied 20 patients who had undergone contrast enhanced computed tomography (EBCT) and 
navigator echo-based coronary MRA with retrospective gating. The results were compared with conventional coronary angiography. The sensitivity and specificity for detecting significant stenoses with coronary MRA were $79 \%$ and $70 \%$, respectively, and with EBCT the sensitivity and specificity were $85 \%$ and $77 \%$, respectively. The low sensitivity with 3D coronary MRA was attributed to inadequate synchronization during the end-expiration phase. EBCT has the advantage of better image quality and less need for patient cooperation, but it also has certain disadvantages, for example, the patient is required to hold his/her breath for approximately 35 seconds. Furthermore, not much progress has been made in the technology, and EBCT is not commonly available in many facilities. Nevertheless, 3D coronary MRA also has several limitations:

1. Some images may not be obtained during the desired end expiratory phase. ${ }^{41}$

2. Patient cooperation is essential with regular, rhythmic respiration and no movement for the whole acquisition time of approximately 35 minutes. ${ }^{41}$

3. Temporal and spatial resolutions are critical factors for image quality in coronary MRA. ${ }^{29,42}$

\section{ADDITIONAL APPLICATION OF CORONARY MRA}

Coronary MRA is a diagnostic modality with excellent soft tissue contrast and, therefore, permits visualization and analysis of plaques and the components of the arteries. This has been shown in the atherosclerotic lesions in vitro and in vivo in large arteries such as the carotid arteries. ${ }^{43,44}$ Coronary MRA is highly effective in detecting coronary artery anomalies and can be regarded as a definite tool for diagnosis. ${ }^{45}$ Conventional coronary MRA is an important diagnostic tool for the diagnosis of patency of coronary artery bypass grafts, as the arteries have lesser mobility with cardiac and respiratory motion and also have a larger lumen $(5-10 \mathrm{~mm}) \cdot{ }^{46}$

Nagel et al. ${ }^{3}$ compared high dose dobutamine stress magnetic resonance imaging (DMRI) with dobutamine stress echocardiography and found that DMRI was more sensitive and more specific than dobutamine stress echocardiography in the detection of myocardial ischemia using coronary stenosis by arteriography as the gold standard. Hundley et al. ${ }^{47}$ reported that DMRI provides excellent diagnostic information as well as prognostic information. Cardiac MRI is ideally suited for the detection of myocardial viability because of its ability to assess the transmurality of myocardial scar, as well as myocardial performance. ${ }^{48}$ At the present state of technical development, the sensitivity and specificity of coronary MRA with retrospective navigator echo respiratory triggering is only modest. The technique is poor and not useful as a screening method for CAD. ${ }^{49}$

\section{CONCLUSION}

Coronary MRA is a rapidly evolving, new non-invasive technique. Although coronary MRA presently has limited clinical utility, it has the potential to aid in the diagnosis of coronary artery stenoses with a high degree of accuracy, especially in the proximal and middle segments, but remains challenging for distal coronary arteries. Magnetic resonance technology has some limitations, which makes it difficult to have better visualization of the coronary arteries. These limitations are secondary to the tortuous course of the coronary arteries, coronary arteries of smaller diameter (2.7 to $3.5 \mathrm{~mm}$ ), rapid movement caused by respiratory and cardiac contractions, and the surrounding epicardial fat.5,50 It may also be difficult to distinguish the coronary arteries from the parallel running coronary veins during the interpretation of coronary MRA, especially for the left circumflex coronary artery. ${ }^{12,18,20,24,25}$

Even though coronary MRA is a potentially useful clinical application, especially in patients with a low probability of CAD, it does not allow visualization of smaller vessels, such as side branches or the distal segments of large epicardial arteries. ${ }^{10}$ Several approaches to coronary MRA have been described, but no one technique can be universally applied. ${ }^{11}$

Future research should focus on the development of optimal respiratory compensation strategies by improving the diagnostic and spatial resolution to visualize greater lengths of coronary arteries and faster acquisition of the data. New strategies are being investigated to improve the diagnostic accuracy, especially with the use of intravenous contrast media, and also to decrease the acquisition time with volume target imaging. ${ }^{51}$ It is conceivable that advances in technology will help in achieving this goal in the not too distant future.

\section{ACKNOWLEDGMENTS}

The authors wish to thank Marshfield Clinic Research Foundation for its support through the assistance of Linda Weis, Jennifer Hayes, and Alice Stargardt in the preparation of this manuscript.

\section{REFERENCES}

1. American Heart Association 2001 heart \& stroke statistical update. Dallas, TX: AHA 2000.

2. Paulin S, von Schulthess GK, Fossel E, Krayenbuehl HP. MR imaging of the aortic root and proximal coronary arteries. AJR Am J Roentgenol 1987;148:665-670.

3. Nagel E, Lehmkuhl HB, Bocksch W, Klein C, Vogel U, Frantz E, Ellmer A, Dreysse S, Fleck E. Noninvasive diagnosis of ischemia-induced wall motion abnormalities with the use of high-dose dobutamine stress MRI: comparison with dobutamine stress echocardiography. Circulation 1999;99:763-770.

4. The clinical role of magnetic resonance in cardiovascular disease. Task Force of the European Society of Cardiology, in collaboration with the Association of European Paediatric Cardiologists. Eur Heart J 1998;19:19-39. 
5. Sechtem U, Baer FM, Voth E, Theissen P, Schneider CA. Stress functional MRI: detection of ischemic heart disease and myocardial viability. J Magn Reson Imaging 1999;10:667-675.

6. van Rossum AC, Galjee MA, Post JC, Visser CA. A practical approach to MRI of coronary artery bypass graft patency and flow. Int J Card Imaging 1997;13:199-204.

7. Toussaint JF, LaMuraglia GM, Southern JF, Fuster V, Kantor HL. Magnetic resonance images lipid, fibrous, calcified, hemorrhagic, and thrombotic components of human atherosclerosis in vivo. Circulation 1996;94:932-938.

8. Fayad ZA. MR imaging for the noninvasive assessment of atherothrombotic plaques. Magn Reson Imaging Clin N Am 2003;11:101-113.

9. Davidson CJ, Fishman RF, Bonow RO. Cardiac catheterization. In: Braunwald E, ed. Heart disease. 5th ed. Philadelphia: W.B. Saunders; 1997. 177-203.

10. Moshage W, Achenbach S, Daniel WG. Novel approaches to the non-invasive diagnosis of coronary-artery disease. Nephrol Dial Transplant 2001;16:21-28.

11. Foo TK, Saranathan M, Hardy CJ, Ho VB. Coronary artery magnetic resonance imaging: a patient-tailored approach. Top Magn Reson Imaging 2000;11:406-416.

12. Danias PG, Edelman RR, Manning WJ. Coronary MR angiography. Cardiol Clin 1998;16:207-225.

13. Dodge JT Jr, Brown BG, Bolson EL, Dodge HT. Lumen diameter of normal human coronary arteries. Influence of age, sex, anatomic variation, and left ventricular hypertrophy or dilation. Circulation 1992;86:232-246.

14. Taylor AM, Jhooti P, Wiesmann F, Keegan J, Firmin DN, Pennell DJ. MR navigator-echo monitoring of temporal changes in diaphragm position: implications for MR coronary angiography. J Magn Reson Imaging 1997;7:629-636.

15. Manning WJ, Edelman RR. Coronary MR angiography. Radiology 1995;195:875-876.

16. Wielopolski PA, Manning WJ, Edelman RR. Single breath-hold volumetric imaging of the heart using magnetization-prepared 3-dimensional segmented echo planar imaging. J Magn Reson Imaging 1995;5:403-409.

17. Edelman RR, Manning WJ, Burstein D, Paulin S. Coronary arteries: breath-hold MR angiography. Radiology 1991;181:641-643.

18. Manning WJ, Li W, Edelman RR. A preliminary report comparing magnetic resonance coronary angiography with conventional angiography. N Engl J Med 1993;328:828-832.

19. Duerinckx AJ, Urman MK. Two-dimensional coronary MR angiography: analysis of initial clinical results. Radiology 1994;193:731-738.

20. Pennell DJ, Bogren HG, Keegan J, Firmin DN, Underwood SR. Assessment of coronary artery stenosis by magnetic resonance imaging. Heart 1996;75:127-133.

21. Post JC, van Rossum AC, Hofman MB, de Cock CC, Valk J, Visser CA. Clinical utility of two-dimensional magnetic resonance angiography in detecting coronary artery disease. Eur Heart J 1997;18:426-433.

22. Watanuki A, Yoshino H, Udagawa H, Yokoyama K, Nitatori T, Hachiya J, Ishikawa K. Quantitative evaluation of coronary stenosis by coronary magnetic resonance angiography. Heart Vessels 2000;15:159-166.

23. Sakuma H, Caputo GR, Steffens JC, O’Sullivan M, Bourne MW, Shimakawa A, Foo TK, Higgins CB. Breath-hold MR cine angiography of coronary arteries in healthy volunteers: value of multiangle oblique imaging planes. AJR Am J Roentgenol 1994;163:533-537.

24. Yoshino H, Nitatori T, Kachi E, Yano K, Taniuchi M, Hachiya J, Ishikawa K. Directed proximal magnetic resonance coronary angiography compared with conventional contrast coronary angiography. Am J Cardiol 1997;80:514-518.
25. Post JC, van Rossum AC, Hofman MB, Valk J, Visser CA. Protocol for two-dimensional magnetic resonance coronary angiography studied in three-dimensional magnetic resonance data sets. Am Heart J 1995;130:167-173.

26. Duerinckx AJ, Atkinson DP, Mintorovitch J, Simonetti OP, Vrman MK. Two-dimensional coronary MRA: limitations and artifacts. Eur Radiol 1996;6:312-325.

27. Paschal CB, Haacke EM, Adler LP. Three-dimensional MR imaging of the coronary arteries: preliminary clinical experience. J Magn Reson Imaging 1993;3:491-500.

28. Li D, Paschal CB, Haacke EM, Adler LP. Coronary arteries: three-dimensional MR imaging with fat saturation and magnetization transfer contrast. Radiology 1993;187:401406.

29. Wielopolski PA, van Geuns RJ, de Feyter PJ, Oudkerk M. Coronary arteries. Eur Radiol 2000;10:12-35.

30. Hofman MB, Paschal CB, Li D, Haacke EM, van Rossum AC, Sprenger M. MRI of coronary arteries: 2D breath-hold vs 3D respiratory-gated acquisition. J Comput Assist Tomogr 1995; 19:56-62.

31. Post JC, van Rossum AC, Hofman MB, Valk J, Visser CA. Three-dimensional respiratory-gated MR angiography of coronary arteries: comparison with conventional coronary angiography. AJR Am J Roentgenol 1996;166:1399-1404.

32. Kim WY, Danias PG, Stuber M, Flamm SD, Plein S, Nagel E, Langerak SE, Weber OM, Pedersen EM, Schmidt M, Botnar RM, Manning WJ. Coronary magnetic resonance angiography for the detection of coronary stenoses. N Engl J Med 2001;345:1863-1869.

33. Muller MF, Fleisch M, Kroeker R, Chatterjee T, Meier B, Vock P. Proximal coronary artery stenosis: three-dimensional MRI with fat saturation and navigator echo. J Magn Reson Imaging 1997; 7:644-651.

34. Kessler W, Achenbach S, Moshage W, Zink D, Kroeker R, Nitz W, Laub G, Bachmann K. Usefulness of respiratory gated magnetic resonance coronary angiography in assessing narrowings $50 \%$ in diameter in native coronary arteries and in aortocoronary bypass conduits. Am J Cardiol 1997;80:989-993.

35. Sandstede JJ, Pabst T, Beer M, Geis N, Kenn W, Neubauer S, Hahn D. Three-dimensional MR coronary angiography using the navigator technique compared with conventional coronary angiography. AJR Am J Roentgenol 1999;172:135139.

36. Sardanelli F, Molinari G, Zandrino F, Balbi M. Three-dimensional, navigator-echo MR coronary angiography in detecting stenoses of the major epicardial vessels, with conventional coronary angiography as the standard of reference. Radiology 2000;214:808-814.

37. Weber C, Steiner P, Sinkus R, Dill T, Bornert P, Adam G. Correlation of $3 \mathrm{D}$ MR coronary angiography with selective coronary angiography: feasibility of the motion-adapted gating technique. Eur Radiol 2002;12:718-726. Epub 2001 Dec 15.

38. Watanabe Y, Nagayama M, Amoh Y, Fujii M, Fuku Y, Okumura A, Van Cauteren M, Stuber M, Dodo Y. High-resolution selective three-dimensional magnetic resonance coronary angiography with navigator-echo technique: segment-by-segment evaluation of coronary artery stenosis. J Magn Reson Imaging 2002; 16:238-245.

39. Regenfus M, Ropers D, Achenbach S, Schlundt C, Kessler W, Laub G, Moshage W, Daniel WG. Comparison of contrast-enhanced breath-hold and free-breathing respiratory-gated imaging in three-dimensional magnetic resonance coronary angiography. Am J Cardiol 2002;90:725-730.

40. Regenfus M, Ropers D, Achenbach S, Kessler W, Laub G, Daniel WG, Moshage W. Noninvasive detection of coronary artery stenosis using contrast-enhanced three-dimensional breath-hold magnetic resonance coronary angiography. J Am Coll Cardiol 2000;36:44-50. 
41. Nikolaou K, Huber A, Knez A, Becker C, Bruening R, Reiser $\mathrm{M}$. Intraindividual comparison of contrast-enhanced electron-beam computed tomography and navigator-echo-based magnetic resonance imaging for noninvasive coronary artery angiography. Eur Radiol 2002;12:1663-1671. Epub 2002 Mar 08.

42. Bunce NH, Pennell DJ. Magnetic resonance of coronary arteries. Eur Radiol 2001;11:721-731.

43. Shinnar M, Fallon JT, Wehrli S, Levin M, Dalmacy D, Fayad ZA, Badimon JJ, Harrington M, Harrington E, Fuster V. The diagnostic accuracy of ex vivo MRI for human atherosclerotic plaque characterization. Arterioscler Thromb Vasc Biol 1999;19:2756-2761.

44. Yuan C, Beach KW, Smith LH Jr, Hatsukami TS. Measurement of atherosclerotic carotid plaque size in vivo using high resolution magnetic resonance imaging. Circulation 1998;98:2666-2671.

45. Vliegen HW, Doornbos J, de Roos A, Jukema JW, Bekedam MA, van der Wall EE. Value of fast gradient echo magnetic resonance angiography as an adjunct to coronary arteriography in detecting and confirming the course of clinically significant coronary artery anomalies. Am J Cardiol 1997;79:773-776.

46. White RD, Caputo GR, Mark AS, Modin GW, Higgins CB. Coronary artery bypass graft patency: noninvasive evaluation with MR imaging. Radiology 1987;164:681-686.

47. Hundley WG, Morgan TM, Neagle CM, Hamilton CA, Rerkpattanapipat P, Link KM. Magnetic resonance imaging determination of cardiac prognosis. Circulation 2002;106:2328-2333.

48. Mankad S, Khalil R, Kramer CM. MRI for the diagnosis of myocardial ischemia and viability. Curr Opin Cardiol 2003;18:351-356.

49. Ikonen AE, Manninen HI, Vainio P, Hirvonen TP, Vanninen RL, Matsi PJ, Soimakallio S, Hartikainen JE. Three-dimensional respiratory-gated coronary MR angiography with reference to x-ray coronary angiography. Acta Radiol 2003;44:583-589.

50. van der Wall EE, Vliegen HW, de Roos A, Bruschke AV. Magnetic resonance imaging in coronary artery disease. Circulation 1995;92:2723-2739.

51. Lorenz CH, Johansson LO. Contrast-enhanced coronary MRA. J Magn Reson Imaging 1999;10:703-708. 